\title{
The ordinary light microscope: an appropriate tool for provisional detection and identification of crystals in synovial fluid
}

\author{
ELISEO PASCUAL, ${ }^{12}$ JUÁN TOVAR, ${ }^{1}$ AND MARÍA TERESA RUIZ \\ From the ${ }^{1}$ Rheumatology Section, Hospital de Alicante; and the ${ }^{2}$ Faculty of Medicine and ${ }^{3}$ Department of \\ Community Medicine, University of Alicante, Alicante, Spain
}

SUMMARY To determine if the ordinary light microscope is a useful tool in the detection and identification of crystals in synovial fluid 63 fluids (13 with monosodium urate (MSU), 14 with calcium pyrophosphate dihydrate (CPPD), one with both types of crystal, and 35 without crystals) were examined blindly by two observers, first with an ordinary light microscope, then with a polarising light microscope. Tentative identification of the crystals by their shape was attempted. The sensitivity of the ordinary light microscope for the detection of crystals was $96.2 \%$ and $100 \%$, with specificity of $100 \%$ and $97.1 \%$ for observers 1 and 2 , both with respect to the polarising light microscope. The concordance between observers for the ordinary light microscope was $x=0.90(0.897,0.902)$ and for the polarising light microscope $x=0.96(0.958$, 0.961). The specificity for identification of MSU and CPPD crystals by their shape with respect to the polarising light microscope was $92.3 \%$ and $85.7 \%$ for observers 1 and 2 . When a polarising light microscope is not available examination of synovial fluid by an ordinary light microscope may allow a provisional diagnosis; definitive identification of the crystals requires a polarising light microscope.

The finding by Dr Joseph Hollander of crystals in the synovial fluid obtained from patients with gout by means of an ordinary light microscope prompted him to show one such preparation to Dr Daniel McCarty. ${ }^{1}$ Dr McCarty's subsequent work identifying the acicular monosodium urate crystals (MSU) and describing the acicular, rhomboidal, and parallelepipedal calcium pyrophosphate dihydrate crystals (CPPD) is widely known ${ }^{2}{ }^{3}$; the optical properties of both types of crystals allow easy identification, and the compensated polarising light microscope has since become the standard tool for crystal identification in all rheumatological units. ${ }^{4-6}$ Unfortunately, a polarising light microscope is not always available and when it is not it may be useful to know the sensitivity and specificity of the widely used ordinary light microscope-with which the original observation was made-for detection and identification of crystals. To determine sensitivity and specificity two different observers analysed

Accepted for publication 27 February 1989.

Correspondence to Dr Eliseo Pascual. Sección de Reumatología. Hospital de Alicante (SVS). Maestro Alonso 109, 03010 Alicante. Spain. blindly a series of synovial fluids with an ordinary light microscope and subsequently with a polarising light microscope. In addition, we attempted to determine the ability of the ordinary light microscope to identify both types of crystal by their shape.

\section{Material and methods}

Sixty three synovial fluids ( 13 with MSU crystals, 14 with CPPD crystals, one with both types of crystal, and 35 without crystals) provided by other rheumatologists were examined blindly in the first hour of their extraction, without knowledge of their origin, by two observers (a rheumatology resident in his last year of training and a rheumatology consultant) with experience in synovial fluid analysis.

The blind synovial fluid analysis began at $400 \times$ with an ordinary light microscope $(10 \times$ eyepiece and $40 \times$ objective) for a minimum of seven minutes and was followed by analysis at $1000 \times(10 \times$ eyepiece and $100 \times$ oil objective) when necessary. The microscope was provided with a halogen lamp and the condenser kept below optimal resolution to enhance refractivity. Crystals were sought, and if 
present, their shape-only acicular or mixed acicular, rhomboidal, and parallelepipedal-was noted. Afterwards the fluids were examined with a polarising light microscope, and a definitive diagnosis of the presence and nature of the crystals was made. At the end of the analysis the results were reported to the patients' doctors and when they did not agree with the original diagnosis a further careful nonblind examination of the synovial fluid was performed for definitive diagnosis.

Table 1 Contingency tables showing the sensitivity and specificity for the detection of the presence of crystals (either type) with the ordinary light microscope (OLM) with respect to the compensated polarised light microscope (PLM) for observers 1 and 2

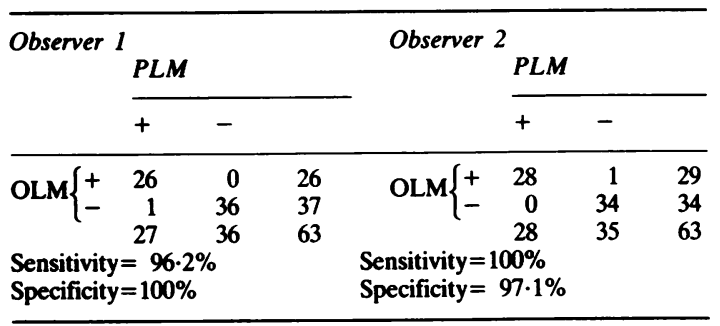

$+=$ presence of crystals; $-=$ absence of crystals.

Table 2 Contingency tables showing the $x$ index of concordance between observers 1 and 2 , for the detection of crystals by the ordinary light microscope and the compensated polarised light microscope

\begin{tabular}{|c|c|c|c|c|c|c|c|}
\hline \multirow[t]{2}{*}{ Ordinary } & \multicolumn{3}{|c|}{$\begin{array}{l}\text { light microscope } \\
\text { Observer } 1\end{array}$} & \multirow[t]{2}{*}{ Polarising } & \multicolumn{3}{|c|}{$\begin{array}{l}\text { light microscope } \\
\text { Observer } 2\end{array}$} \\
\hline & + & - & & & + & - & \\
\hline $\begin{array}{l}\text { Obs } 2\left\{\frac{+}{-}\right. \\
x=0.90\end{array}$ & $\begin{array}{r}26 \\
3 \\
29 \\
0.897\end{array}$ & $\begin{array}{r}0 \\
34 \\
34 \\
.902)\end{array}$ & $\begin{array}{l}26 \\
37 \\
63\end{array}$ & $\begin{array}{l}\text { Obs } 2\left\{\begin{array}{l}+ \\
-\end{array}\right. \\
=0.96(0.9\end{array}$ & $\begin{array}{r}27 \\
1 \\
28 \\
58,0\end{array}$ & $\begin{array}{r}0 \\
35 \\
35\end{array}$ & $\begin{array}{l}27 \\
36 \\
63\end{array}$ \\
\hline
\end{tabular}

$+=$ presence of crystals (either type); - =absence of crystals.
STATISTICAL ANALYSIS

Appropriate tests were used for specificity and sensitivity calculations. ${ }^{7}$ The $x$ test was used for the analysis of concordance; the degree of concordance was expressed as a numerical value for $x$ which can range from $-1 \cdot 0$, indicating absolute discordance, to $1 \cdot 0$, indicating perfect concordance. A completely random result produces a value for $x$ of 0 . A value for $x$ above 0.75 indicates important concordance. ${ }^{8} \stackrel{\infty}{\infty}$

\section{Results}

DETECTION OF CRYSTALS

With the ordinary light microscope observer 1 found crystals in 26 of 27 synovial fluids in which crystals were truly present-that is a sensitivity of $96 \cdot 2 \% \stackrel{\circ}{\oplus}$ with specificity of $100 \%$, both with respect to the $\vec{N}$ polarising light microscope. Observer 2 found crys- 0 tals in 28 synovial fluids with the ordinary light $\infty$ microscope, but analysis with the polarising light 을 microscope showed that there was a false positive for the ordinary light microscope; for this observer $\square$ the sensitivity was $100 \%$ and the specificity $97 \%$ (Table 1). One synovial fluid, which was later found $\frac{\mathbb{}}{3}$ to have MSU crystals, was interpreted as lacking crystals by both the ordinary and polarising light microscopes and by both observers, and this flued was not included in the calculations.

COMPARISON OF THE ORDINARY AND POLARISING LIGHT MICROSCOPES

With the ordinary light microscope observer 1 saw crystals in three synovial fluids (one of them a false $\frac{\%}{\mathbb{Q}}$ positive finding), which were not seen by observer $2 ; \stackrel{2}{?}$ the $x$ index for concordance between observers for $\overrightarrow{0}$ this microscope was $x=0.90(0.897,0.902)$. With the polarising light microscope there was discordance in only one synovial fluid, which was wrongly inter-? preted as having crystals by observer 1 ; the $x$ index for this microscope was $x=0.96(0.958,0.961) \frac{2}{\sigma}$ (Table 2).

IDENTIFICATION OF CRYSTAL TYPE

For this analysis the fluid containing both MSU and

Table 3 Contingency tables showing the specificity for the identification of crystals by their shape with the ordinary light microscope $(O L M)$ with respect to the compensated polarised light microscope (PLM), by observers 1 and 2

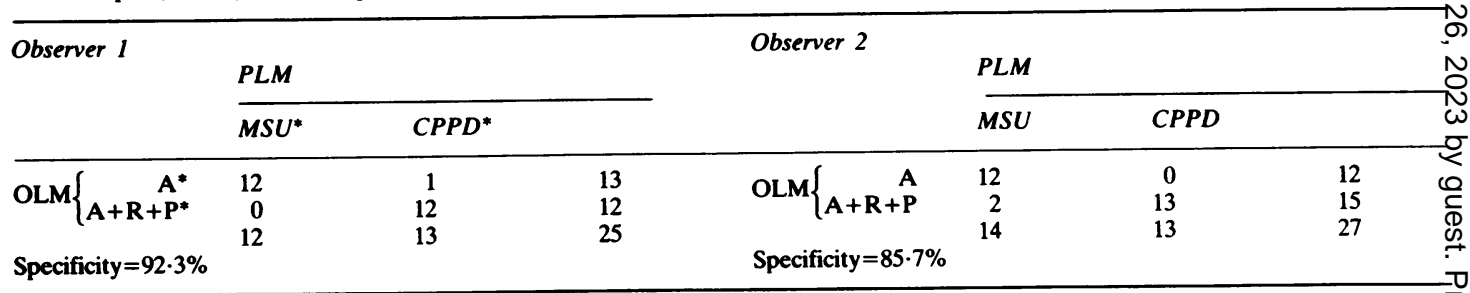

*A+R+P=acicular, rhomboidal, and parallelepipedal crystals; $A=$ only acicular crystals; $M S U=$ monosodium urate crystals; $C P P D=0$ calcium pyrophosphate dihydrate crystals. 
CPPD crystals was not considered. Of the additional 25 fluids in which observer 1 saw crystals with the ordinary light microscope, only one was wrongly interpreted as MSU when it was really CPPD; this gave a specificity of $92.3 \%$ with respect to the polarising light microscope. Observer 2 with the ordinary light microscope interpreted two synovial fluids as containing CPPD, which the polarising light microscope identified as MSU; this gave a specificity of $85.7 \%$ (Table 3 ). In the synovial fluid which contained both types of crystals both observers made an accurate diagnosis with the ordinary light microscope.

\section{Discussion}

The ability of the ordinary light microscope to detect MSU or CPPD crystals in synovial fluid is known, ${ }^{4}$ but the practical consequences of this have not been explored. The results of this work show that the ordinary light microscope, used by trained observers, is a sensitive tool for detecting the presence of crystals (either MSU or CPPD) in synovial fluid; only one of the observers missed their presence in one of the 27 fluids which contained them. In addition, the specificity of the ordinary light microscope for the detection of crystals, with respect to the polarising light microscope, is high, and on only one occasion was a structure floating in the synovial fluid interpreted as being a crystal, giving a 'false positive'. The concordance for detection of crystals between the observers with the ordinary light microscope was only slightly lower than that found with the polarising light microscope, and both were excellent. In one fluid which contained MSU crystals their presence was missed in the blind study with both microscopes and by both observers. Once its origin was known centrifugation allowed detection of the crystals in the pellet.

The high specificity found for the ordinary light microscope, with respect to the polarising light microscope, in the identification of crystals by their shape is of additional interest; those synovial fluids containing only acicular crystals were likely to contain MSU, whereas the synovial fluids with parallelepipedal and rhomboidal as well as acicular structures were most likely to contain CPPD crystals. The acicular crystals of CPPD have clearly defined tips, whereas the tips of the MSU crystals are often less well defined and seem to fan. This different appearance becomes clear with practice, and allowed both observers using the ordinary light microscope to note that one of the synovial fluids had both types of crystal. CPPD crystals are weakly birefringent with the polarising light microscope and at times nearly isotropic. ${ }^{6}$ It has been our experience that this type of crystal is often better seen by the ordinary light microscope, and that when the crystals were small observation at $1000 \times$ helped to distinguish between them and other intracellular inclusions.

The polarising light microscope is the appropriate tool for detecting and identifying crystals in synovial fluid $^{4-6}$; to fit an ordinary light microscope with polarising lenses is very simple and cheap ${ }^{9}$ and allows proper identification of the usual crystals. Despite this a polarising light microscope may not be available and then our results suggest that an examination of the synovial fluid with an ordinary light microscope is useful. A trained observer with an ordinary light microscope has a good chance of detecting crystals if present, and even identifying them, thus providing an insight into the nature of an acute arthritis and initial guidance for its treatment. It is not the purpose of this paper to encourage the widespread use of the ordinary light microscope for detection and identification of crystals in synovial fluid, and untrained personnel should be cautioned against it. It needs to be stressed that the diagnosis of crystal induced synovitis made with an ordinary light microscope can only be tentative, and that definitive confirmation of the presence of crystals and identification of their nature with polarising light microscope must always be carried out.

\section{References}

1 McCarty D J. Crystal deposition diseases. In: McCarty D J, ed. Landmark advances in rheumatology. Atlanta: Arthritis Rheumatism Association, 1985: 73-88.

2 McCarty D J, Hollander J L. Identification of gouty crystals in gouty synovial fluid. Ann Intern Med 1961; 54: 452-60.

3 Kohn N N, Hughes R E, McCarty D J Jr, Faires J S. The significance of calcium pyrophosphate crystals in the synovial fluid of arthritis patients: the "pseudogout syndrome". II. Identification of crystals. Ann Intern Med 1962; 56: 738-45.

4 Schumacher H R. Synovial fluid analysis. In: Kelley W N, Harris E D, Ruddy S, Sledge C B, eds. Textbook of rheumatology. 2nd ed. Philadelphia: Saunders, 1985: 561-7.

5 McCarty D J. Synovial fluid. In: McCarty D J, ed. Arthritis and allied conditions. 10th ed. Philadelphia: Lea and Febiger, 1985: $54-75$.

6 Gatter R A. A practical handbook of joint fluid analysis. Philadephia: Lea and Febiger, 1984: 37-49.

7 Fletcher R H, Fletcher S W, Wagner E H. Clinical epidemiology: the essentials. Baltimore: Williams and Wilkins, 1982: 41-58.

8 Fleiss J L. Statistical methods for rates and proportions. 2nd ed. New York: Wiley, 1981: 212-36.

9 Owen D S. A cheap and useful compensated polarizing microscope. N Engl J Med 1971; 285: 1152. 